\title{
Virtuelle Produktentwicklung mittels Simulationsmethoden und KI
}

\author{
Gestiegene Materialvielfalt und höhere Bauteilanforderungen bei gleichzeitig \\ kürzer werdenden Entwicklungszyklen erhöhen Komplexität und Entwick- \\ lungsaufwand aktueller Leichtbaulösungen. Wissenschaftler am KIT \\ untersuchen, wie zukünftig Simulationen und maschinelles Lernen \\ kombiniert werden können, um Entwicklungsprozesse zu beschleunigen.
}

Seit vielen Jahrzehnten ermöglichen Forschung und Entwicklung immer leistungsfähigere Material- und Konstruktionslösungen mit Faser-Kunststoff-Verbunden (FKV). Dem erreichbaren Leichtbaugrad steht bisher jedoch ein hoher Aufwand zur fertigungsgerechten Bauteilgestaltung und Prozesseinrichtung gegenüber. Nur mit effizienten Auslegungsmethoden kann das hohe Leichtbaupotenzial der FKV im finalen Produkt wirtschaftlich umgesetzt werden und so höhere Werkstoffkosten rechtfertigen. Dies gilt besonders im Hinblick auf Mittel- und Großserienprozesse, bei denen die Interaktion zwischen Bauteilperformance und ökonomischen Anforderungen besonders ausgeprägt ist, Bild 1 (links).
Durch den wachsenden Einsatz von FKV und zahlreiche Forschungsergebnisse der letzten Jahrzehnte hat sich in Industrie und Forschung ein fundiertes Material-, Prozessund Simulationsverständnis etabliert $[1,2]$. Dabei wird ein vollständiges Ausschöpfen vorhandener Leichtbaupotenziale erst durch eine optimale Abstimmung von Werkstoff, qualitätsgerechter Fertigung und systemgerechter Konstruktion erreicht - eine herausfordernde Optimierungsaufgabe. Klassische Entwicklungsprozesse beinhalten zahlreiche experimentelle Aufwände, Konzeptvarianten und -überarbeitungen, Iterationen und gegebenenfalls Neustarts. Eine durchgängig digitalisierte Produktentwicklung bietet weitreichende Chancen, das Leichtbaupotenzial, die Wirtschaftlichkeit und die Nachhaltigkeit innerhalb von Entwicklungsprozessen im Leichtbau zu steigern. Zur Unterstützung der Produktentwicklung kann die virtuelle Prozesskette mit Echtzeitdaten und Methoden des Maschinenlernens (ML) ergänzt werden.

\section{Die durchgängig virtuelle Prozesskette}

Eine virtuelle Prozesskette ermöglicht die direkte Verknüpfung von Design und finalem FKV-Bauteil unter Berücksichtigung der Wechselwirkungen zwischen Material und 


\section{Autoren}

DIPL.-ING. CLEMENS

ZIMMERLING

ist Wissenschaftlicher

Mitarbeiter im Bereich

Prozess- und Struktur-

optimierung für Leicht-

bautechnologien am

Institut für Fahrzeug-

systemtechnik (FAST)

des Karlsruher Instituts

für Technologie (KIT) in

Karlsruhe.

CHRISTIAN POPPE,

M. SC.,

ist Wissenschaftlicher

Mitarbeiter im Bereich

Prozesssimulation für

Leichtbautechnologien

am Institut für Fahr-

zeugsystemtechnik

(FAST) des Karlsruher

Instituts für Technolo-

gie (KIT) in Karlsruhe.

DR.-ING. LUISE KÄRGER

ist Stellvertretende

Leiterin des Teilinsti-

tuts für Leichtbautech-

nologie am Institut für

Fahrzeugsystemtechnik

(FAST) des Karlsruher

Instituts für Technolo-

gie (KIT) in Karlsruhe.
Prozess bei der Herstellung. Beispielsweise prägen sich Eigenspannungen infolge chemo-thermomechanischer Effekte während der Aushärtung und Abkühlung aus. Sie können die Tragfähigkeit einer Struktur signifikant beeinflussen und sind daher in einer gekoppelten Prozess- und Bauteilauslegung zu berücksichtigen. Zudem beeinflusst bei heterogenen Werkstoffen wie FKV die prozessbedingte Mikrostruktur entscheidend das letztliche Bauteilverhalten.

Die physikalischen Wechselwirkungen zwischen Material und Prozess sind mittels gleichzeitig stetig kürzer werdenden Entwicklungs- und Produktionszyklen erhöhen den Entwicklungsaufwand im hybriden Leichtbau. Belastbare virtuelle Vorhersagen der material- und prozessspezifischen Wechselwirkungen sind für ein grundlegendes Systemverständnis und eine sichere und effiziente Auslegung unerlässlich. Unzureichende Modellierungsansätze können zu fehlerhaften Aussagen führen. Insbesondere wenn eine hohe Auslastung von Material, Prozess oder Struktur gewünscht ist, bleibt wenig Raum für Ungenauigkeiten. Die fol-

\section{Virtuelle Vorhersagen der material- und prozessspezifischen Wechselwirkungen sind unerlässlich.}

skalenübergreifender und Multiphysikbasierter Simulationsansätze zu beschreiben und innerhalb durchgängiger virtueller CAE-Ketten, Bild 1 (rechts) miteinander zu koppeln. Die Schaffung standardisierter und neutraler Austauschformate zwischen verschiedenen Simulationscodes, wie etwa im ITEA-Projekt VMAP, trägt dazu bei, anwenderspezifische Insellösungen zu vermeiden.

\section{Multiphysikalische Wechselwirkungen}

Gestiegene Anforderungen an Zuverlässigkeit und Komplexität von Bauteilen bei genden Beispiele illustrieren, dass die Berechnungsmethodik entscheidend ist, um Material- und Fertigungseffekte sowie die resultierende Tragfähigkeit zuverlässig vorherzusagen.

\section{Lokale Entmischungseffekte an Rippen}

Bei der Herstellung von langfaserverstärkten Sheet-Molding-Compound(SMC)-Bauteilen kann es an engen Querschnitten und Bindenähten zu einer lokalen Entmischung von Fasern und Matrix kommen Bild 2 (links). Herkömmliche, homogenisierte Simulationsmodelle nutzen den Faserorientierungs-

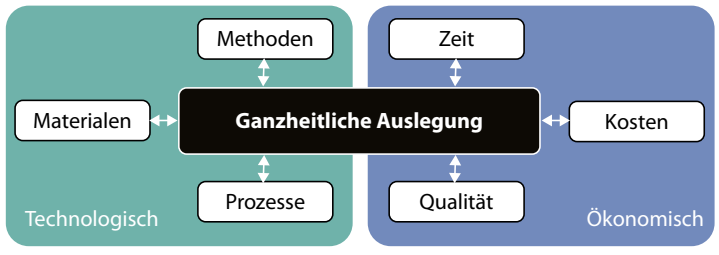

Anforderungen an wirtschaftliche Leichtbaulösungen

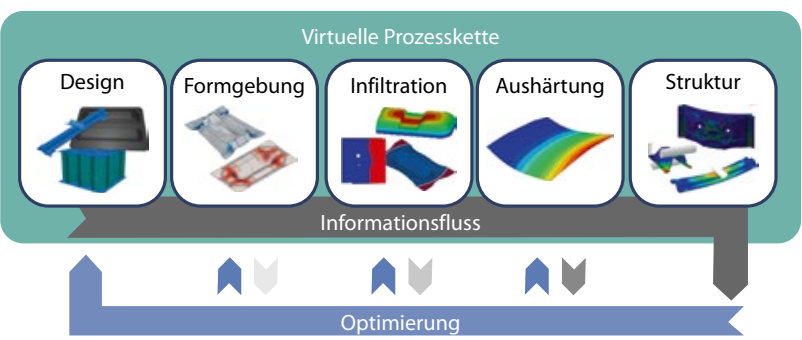

Umsetzung innerhalb einer virtuellen Prozesskette

Bild 1 Technologische und ökonomische Anforderungen an eine effiziente FKV-Lösung [1] (links) und Beispiel einer durchgängigen virtuellen CAE-Prozesskette [2] (rechts) (๑ KIT|FAST) 


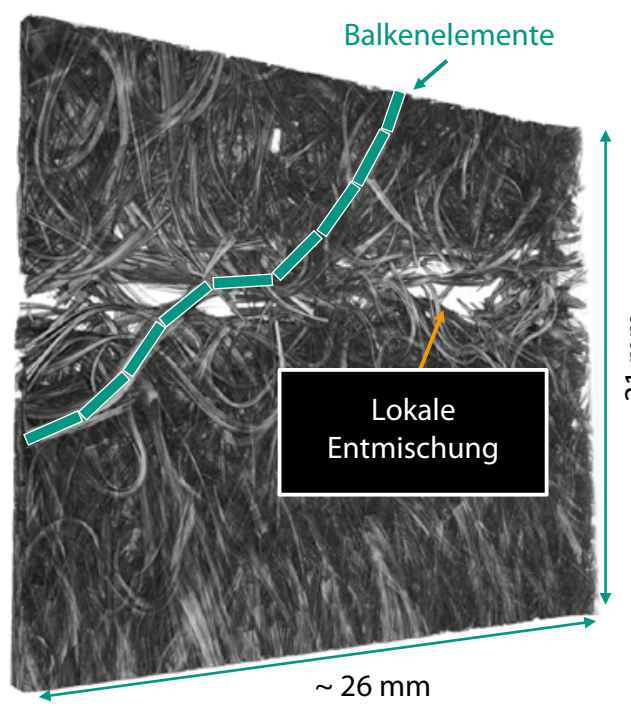

SMC-Mesostruktur im CT

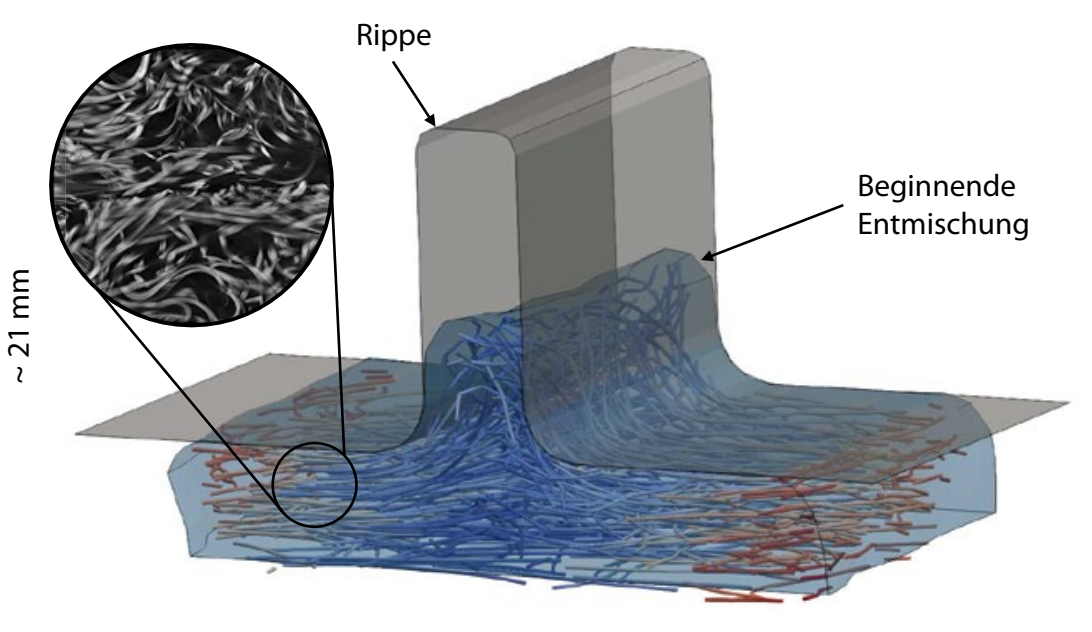

Simulationsmodell

Bild 2 Mesostruktur eines SMC-Probekörpers im CT-Scan (links) und Einströmsimulation des Matrixmaterials mit eingebetteten Faserbündeln in eine Rippe während des Fließpressens [3] (rechts) (@ KIT|FAST)

tensor zur Beschreibung der Materialanisotropie und anderer Effekte auf der Mesoskala. Entmischungsvorgänge können diese Ansätze nicht abbilden. Bei einer klassischen Auslegung mit homogenisierten Materialkennwerten würde dies zu einer Überschätzung der Rippensteifigkeit führen.

Eine vielversprechende Lösung hierfür ist die explizite Modellierung der defor- mierbaren Faserbündel Bild 2 (rechts) inklusive ihrer Wechselwirkung mit den hydrodynamischen Fluidkräften des Strömungsfeldes der Matrix [3]. Somit erfolgt eine direkte Berücksichtigung der auftretenden Fluid-Struktur-Interaktion. Die direkte Simulation der Bündel benötigt keine Parameter- und Schließungsansätze zur Bestimmung der Faserorientierung. Des Weiteren umfasst der gekoppelte Ansatz das anisotrope Fließverhalten der Fasersuspension. Entwickelt wird diese Methode aktuell in einem Forschungsprojekt im Rahmen des DFG-Graduiertenkollegs GRK 2078.

Der neue Simulationsansatz ermöglicht es, den Einfluss relevanter Prozessparameter wie Werkzeugbelegung, -schließprofil oder der Rippengeometrie auf die Mesostruktur

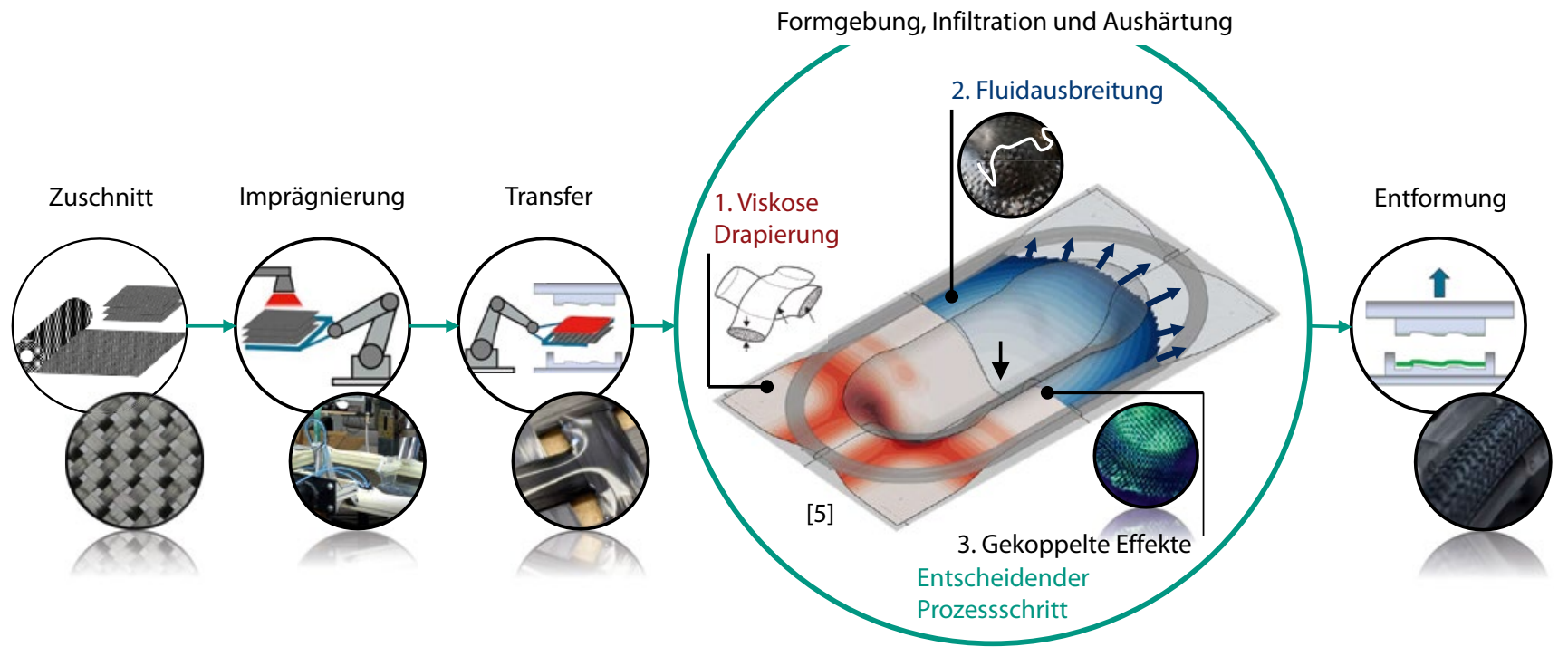

Bild 3 Darstellung der Nasspressprozesskette vom Zuschnitt bis zur Entformung mit besonderem Fokus auf die Simulation der simultanen Formgebung (Scherwinkel in rot) und Infiltration (Druckverteilung in blau) [4] (๑ KIT|FAST und Fraunhofer ICT) 
im finalen Bauteil zu untersuchen und bei der strukturellen Auslegung korrekt zu berücksichtigen.

\section{Formgebung und Infiltration im Nasspressprozess}

Komplexe Wechselwirkungen zwischen Material und Prozess sind charakteristisch für den hybriden Leichtbau, so auch beim Nasspressen. Bei diesem Verfahren, das in der automobilen Großserie als Alternative zum Resin Transfer Moulding (RTM) eingesetzt wird, werden Formgebung (Drapierung), Formfüllung (Infiltration) und Aushärtung in einem Prozessschritt realisiert, Bild 3. Auf diese Weise können gegenüber dem herkömmlichen RTM-Prozess ein Prozessschritt eingespart und Taktzeiten im Bereich 60 bis 90 s erreicht werden.

Die zentralen Prozessdynamiken beim Nasspressen lassen sich nur mit einem kombinierten Simulationsansatz für die Drapierung und Formfüllung beschreiben [4]. Gegenwärtige Modelle bilden diese Multiphysikeffekte nicht ab, weshalb eine konstruktive Auslegung der Werkzeug- und Dichtkonzepte momentan nicht virtuell unterstützt wird.
Im Projekt „Forschungsbrücke“ werden die physikalischen Wirkmechanismen und Prozessgrenzen der Nasspresstechnologie anhand endlosfaserverstärkter Bauteile in Kooperation zwischen dem KIT-FAST, dem IFB Stuttgart und dem Fraunhofer ICT untersucht. Die Herausforderung liegt darin, die oben beschriebenen Wechselwirkungen in einem effizienten numerischen Modell zu beschreiben. Die zentralen Mechanismen beim Nasspressen sind:

- Viskose Drapierung der Halbzeugschichten

- Simultan ablaufende Fluidausbreitung und Aushärtung

- Fluid-Struktur-Interaktion (FSI) zwischen Faserhalbzeugen und Matrix.

Gerade dieses untrennbare Zusammenspiel der Effekte ist jedoch von entscheidender Bedeutung für die finale Bauteilqualität [5]. Eine isolierte Betrachtung und Optimierung einzelner Effekte für die Prozessauslegung ist nicht möglich.

Aus diesem Grund werden Modellierungsansätze verfolgt, die eine gleichzeitige Abbildung der auftretenden Effekte erlauben, hier im Speziellen Formgebung und Infiltration [4]. Aktuell wird an einem drei- dimensionalen Ansatz geforscht, der die Vorhersage messbarer Prozessgrößen wie Kavitätsinnendrücke und qualitätsrelevante Fertigungseffekte - zum Beispiel Trockenstellen oder Faserauswaschungen ermöglicht.

\section{Fertigungsbedingte Imperfektionen}

Einflüsse der Fertigung auf das Bauteil sind unvermeidlich. Ihr Einfluss auf die Tragfähigkeit der Struktur ist daher in der Auslegung zu berücksichtigen. Dies ermöglicht zudem, Konstruktionsprinzipien zu verwenden, die fertigungsbedingte Imperfektionen des Materials wie zum Beispiel Faserwelligkeiten, Lückenbildung oder Rovingkompaktierungen berücksichtigen, Bild 4. Hierfür sind Informationen aus vorangehenden Prozessschritten essenziell. Um unter den Effekten tragrelevante Defekte zu erkennen, müssen diese im Materialverhalten der Struktursimulation beachtet werden.

Klassische Konstruktionsvorschriften gehen von einer defektfreien, idealisierten Faserverstärkung in der Matrix aus und überschätzen daher häufig die Werkstoff-

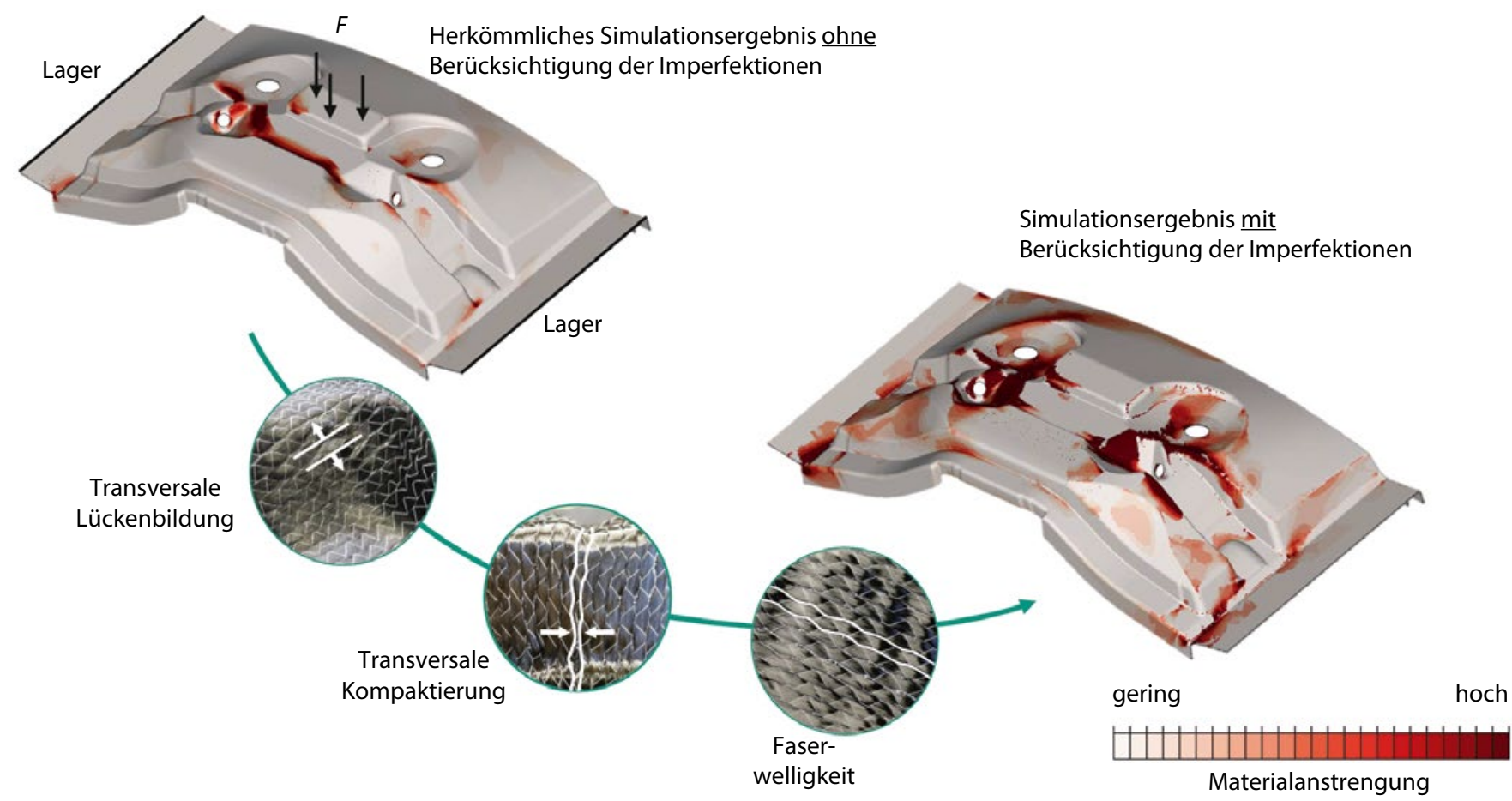

Bild 4 Beispiel für den Einfluss fertigungsbedingter Imperfektionen auf die Vorhersage der Materialanstrengung bezogen auf Zwischenfaserbrüche (@ KIT|FAST) 
leistungsfähigkeit. Ziel eines von der DFG geförderten Forschungsprojekts ist es, solche Fertigungseffekte skalenübergreifend beschreiben, quantifizieren und bei der Auslegung von Bauteilen aus FKV berücksichtigen zu können [6]. Bild 4 zeigt, dass potenzielle Zwischenfaserbrüche stärker und genauer aufgelöst vorhergesagt werden. Wenn neben den lokalen Faserorientierungen auch die Imperfektionen korrekt übertragen werden, unterscheiden sich nicht nur die Orte erhöhter Materialanstrengung, sondern auch ihre Ausprägung an einigen Stellen um mehr als $60 \%$. Solche hochaufgelös-
Material- und Prozesseffekte zu frühen Zeitpunkten der Entwicklung kann das volle Potenzial kommender Leichtbaulösungen erschlossen werden.

Mit den gestiegenen Anforderungen an Simulationstechnik und -expertise ergeben sich auch neue Hürden für die Produktentwicklung. Einerseits ist der Zugang zu leistungsfähiger Rechentechnik und teurer Simulationssoftware eine finanzielle Barriere, insbesondere für kleine und mittelständische Unternehmen (KMU). Andererseits ist die Kombination aus wachsender Materialvielfalt, mehrstufigen Prozessen

\section{Maschinenlernen}

Unter dem Begriff Maschinenlernen werden Techniken der künstlichen Intelligenz zusammengefasst, die eine Software oder eine Maschine dazu befähigen, eine vorab definierte Aufgabe zu erfüllen ohne jedoch explizit für diese Aufgabe von außen programmiert zu werden. Die Maschine muss die Strategien zur Lösung der Aufgabe selbstständig aus bereitgestellten Daten extrahieren. [7]

\section{Potenzielle Zwischenfaserbrüche können durch die Berücksichtigung von Fertigungs- effekten genauer vorhergesagt werden.}

ten Ansätze ermöglichen eine fundierte Bewertung der Folgen von Imperfektionen in der finalen Bauteilstruktur.

\section{Komplexitäten organisieren und zugänglich machen}

Die Entwicklungsbeispiele aus der Forschung verdeutlichen, dass künftige Leichtbaulösungen maßgeblich von einer verbesserten Abstimmung zwischen Material, Prozess und Strukturanforderung profitieren. Nur mit einem kontinuierlichen Informationsfluss zur Berücksichtigung spezifischer und erheblichen Strukturanforderungen ein herausfordernder Optimierungsraum mit komplexen Abhängigkeiten. Unter diesen Bedingungen stoßen klassische Verfahren der mathematischen Optimierung rasch an Grenzen: Sie verlieren sich häufig in lokalen Optima oder benötigen zahlreiche Iterationen, was die Gesamtberechnungszeit immens erhöht - nicht selten in der Größenordnung von Tagen bis Wochen.

In der Praxis greifen Unternehmen daher häufig auf Best-practice-Leitfäden und Mitarbeitererfahrung zurück. Solches Vorwissen zum Material- oder Prozessverhalten hilft, die Variantenvielfalt bereits vorab auf technisch vielversprechende, aber nicht zwingend fehlerfreie Optionen einzuschränken. Häufig entstehen hierbei signifikante Kosten in der Fehlerkorrektur, Feinabstimmung und Nacharbeit.

Wünschenswert ist daher, die Reproduzierbarkeit und Verlässlichkeit von Simulationsmethoden mit dem physikalischen Verständnis und technischen Vorwissen eines Ingenieurs zu verbinden. Eine aussichtsreiche Möglichkeit hierzu bieten Verfahren des Maschinenlernens (ML). Die Idee ist, MLAlgorithmen so mit Simulationen zu koppeln, dass sie den Optimierer im Parameterraum leiten und damit die Entwicklung insgesamt beschleunigen.

\section{Neuronale Netze als Hilfsmittel}

Unter dem Begriff ML werden allgemein Methoden zusammengefasst, die Zusam-
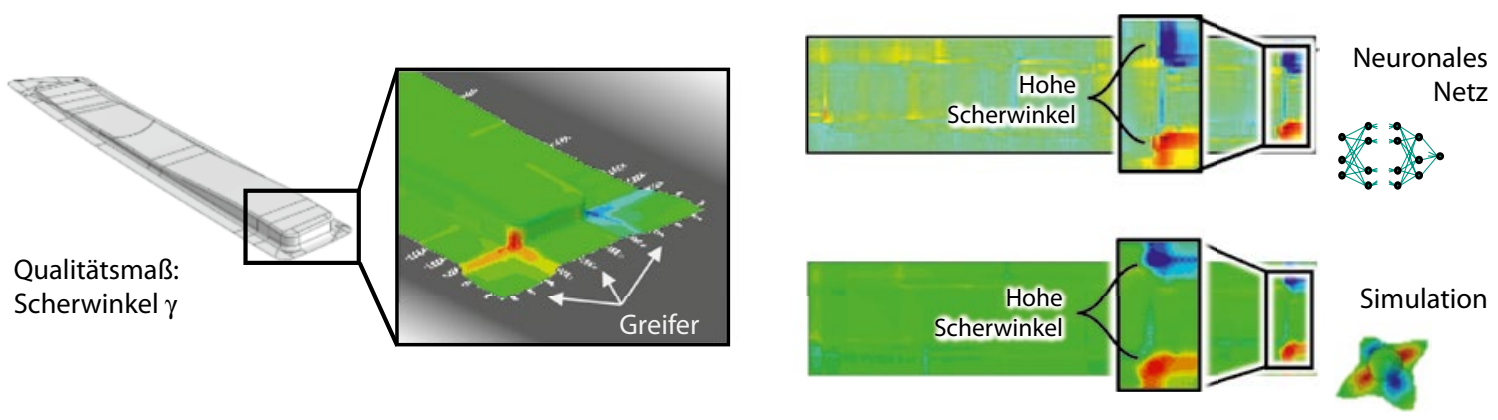

Bild 5 schema der greiferunterstützten Textilumformung (links) und Vergleich der Scherwinkel in Abhängigkeit der Greiferkräfte nach ML-Schätzung und Simulation (rechts) [8] (๑ KIT|FAST) 

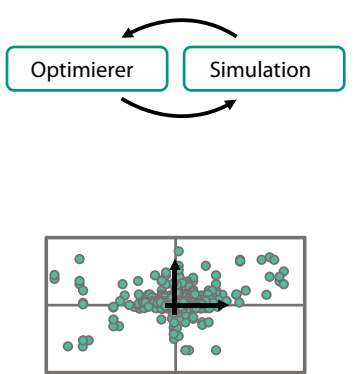

Variation der Auftragsorte

Direkte Optimierung: Starke Streuung im Parameterraum

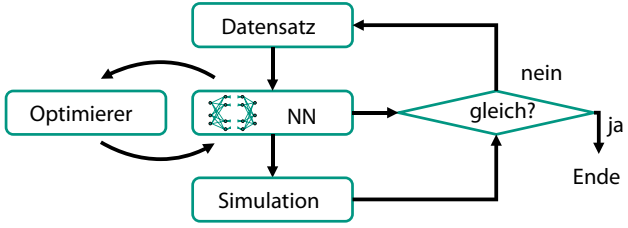

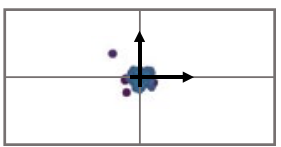

Auftragsorte (Beginn)

ML-unterstützte Optimierung: Simulationen konzentrieren sich im meistversprechenden Bereich (Bauteilmitte)

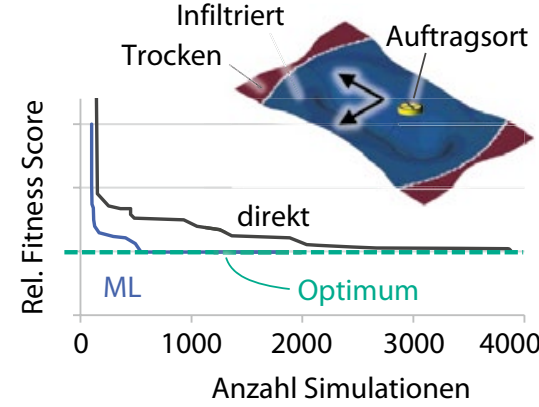

Konvergenzvergleich:

ML-gestützte Optimierung konvergiert schneller zum Optimum

Bild 6 schema und Konvergenzvergleich zwischen direkter und ML-unterstützter Optimierung beim Nasspressen (@ KIT|FAST)

menhänge aus Datensätzen extrahieren, um Vorhersagen für neue Situationen geben zu können. Hierzu zählen verschiedene Techniken, beispielsweise Polynomregression, Gaußprozessregression oder künstliche neuronale Netze. Als Teil des Projekts „Forschungsbrücke" untersucht das KIT-FAST am Beispiel des Nasspressens, inwiefern ML-Verfahren die Entwicklung komplexer Prozesse unterstützen können.

Voruntersuchungen zeigen, dass tiefe künstliche neuronale Netze $(\mathrm{NN})$ besonders geeignet sind, um die in Prozessen auftretenden komplexen Wechselwirkungen zu lernen [8]. So zeigen sie nicht nur eine verbesserte Vorhersagegüte im Vergleich zu anderen ML-Techniken, sondern ermöglichen es auch, Fertigungseffekte lokal aufzulösen, Bild 5. Dies erleichtert die Bewertung eines Prozessergebnisses durch den Ingenieur und die Ableitung geeigneter Handlungsoptionen.

\section{Integration von "virtuellem Vorwissen" in die Optimierung} Direkte Kopplungen von Optimierern mit einem Simulationsmodell führen meist zu einem breit gestreuten Absuchen des Parameterraums, Bild 6 (links). Zur Beschleunigung können ML-Modelle eingesetzt werden, um ungünstige Varianten vorab auszuschließen und die zeitaufwendigen Simula- tionen auf die meistversprechenden Bereiche zu konzentrieren.

Hierzu werden neuronale Netze mit Beispielsimulationen vortrainiert, um anschließend das Ergebnis einer neuen Prozessvariante in wenigen Sekunden abschätzen zu können, so zum Beispiel in die Formfüllzeit je Harzauftragsort. Eine Optimierung auf dem neuronalen Netz läuft dann in wenigen Minuten ab und liefert so eine erste Prozessempfehlung. Durch die gezielte Zwischen- den zu einem früheren Zeitpunkt in den Entwicklungsprozess zu integrieren. Forschungsergebnisse zeigen, dass neuronale Netze aus der Bilderkennung („,neuronale Faltungsnetze") lernen können, die Geometrie eines Bauteils zu interpretieren und bezüglich der Herstellbarkeit zu bewerten, Bild 7 (links). Anhand eines umfangreichen Datensatzes aus Prozesssimulationen, hier Umformsimulationen, unterschiedlicher Geometrien, Bild 7 (rechts) lernt ein

\section{Anwendungsabhängig können 30 bis $70 \%$ der Simulationen eingespart werden.}

schaltung von Simulationen lernt das Netz sukzessive seine Prognosegüte zu verbessern - Prozessempfehlungen werden zuverlässiger, Bild 6 (Mitte). Anwendungsabhängig können 30 bis $70 \%$ der Simulationen eingespart werden, Bild 6 (rechts).

\section{Geometriebewertung durch Maschinenlernen}

Gegenwärtige Forschungsarbeiten zielen darauf ab, die Fähigkeiten von ML-Metho-
Algorithmus, welche Bauteilmerkmale umformrelevant sind. Auf dieser Basis kann er anschließend die Umformbarkeit eines neuen Bauteilentwurfs schnell bewerten und potenziell kritische Stellen identifizieren [9].

Vergleichbar einem „virtuellen Prozessexperten“ kann so ein Algorithmus dem Konstrukteur schnell Feedback zum Bauteilentwurf geben - ohne neuen Simulationsaufwand gegenüber dem Training, reproduzierbar und portabel als Software. Gekoppelt 


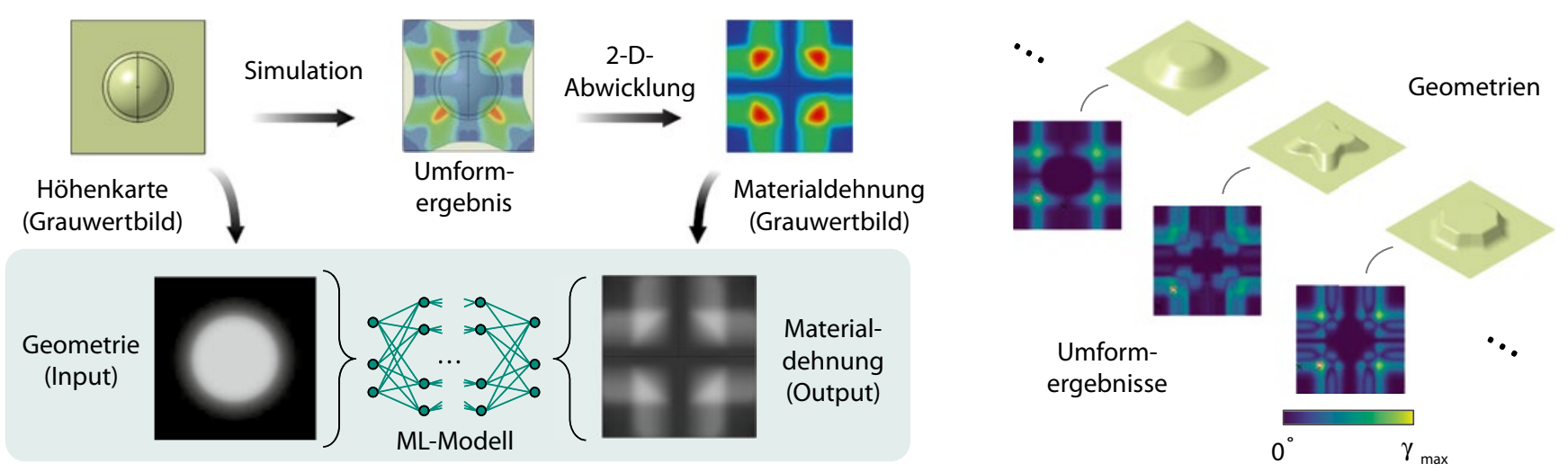

BILD 7 Bildbasierte Datenaufbereitung für neuronale Faltungsnetze (links) und beispielhafte Geometrien der Geometrie-Prozessergebnis-Trainingsdaten (rechts) [9] (๑ KIT|FAST)

mit Optimierungsalgorithmen sind rasche Geometrieanpassungen zur Verbesserung der Drapierbarkeit möglich [10].

\section{Ideengeber und Assistent KI}

Weiterführende Ideen beschreiben den Übergang von einem passiv-reaktiven Feedback-Tool zu einem pro-aktiven Berater: Jüngste Entwicklungen deuten darauf hin, dass fortgeschrittene KI-Techniken fähig sind, aktiv Vorschläge zur Material-, Bauteiloder Prozesskonfiguration zu liefern, wenn Ihnen Randbedingungen, etwa Bauraumoder Lastvorgaben, genannt werden [10, 11]. Dabei ist es den Algorithmen möglich, eine Menge an Varianten zu vergleichen, die ein Mensch nicht mehr bearbeiten kann. Die KIgenerierten Vorschläge können Ingenieure bewerten, sinnvoll zusammenzuführen oder als allgemeine Anregung nutzen, um im Parameter- und Variantenraum Orientierung zu finden.

Unterstützt durch solche Tools können Ingenieure von Routineaufgaben, etwa wiederkehrende Konstruktionsaufgaben
Beispielsweise könnten sich Simulationsspezialisten stärker auf die Weiterentwicklung von Berechnungsmethoden konzentrieren, während ihre Methoden mithilfe von KI einem breiten Anwenderfeld zugänglich gemacht werden. Gegenwärtig sind solche Werkzeuge in der Entstehung

\section{KI-Techniken liefern aktiv Vorschläge zur Material-, Bauteil- oder Prozesskonfiguration.}

oder Materialvariationen, befreit werden und sich stattdessen auf wertschöpfende und kreative Tätigkeiten konzentrieren. und Erprobung und konzentrieren sich auf einfache Anwendungsfälle. Doch insgesamt zeichnen sich perspektivisch neue

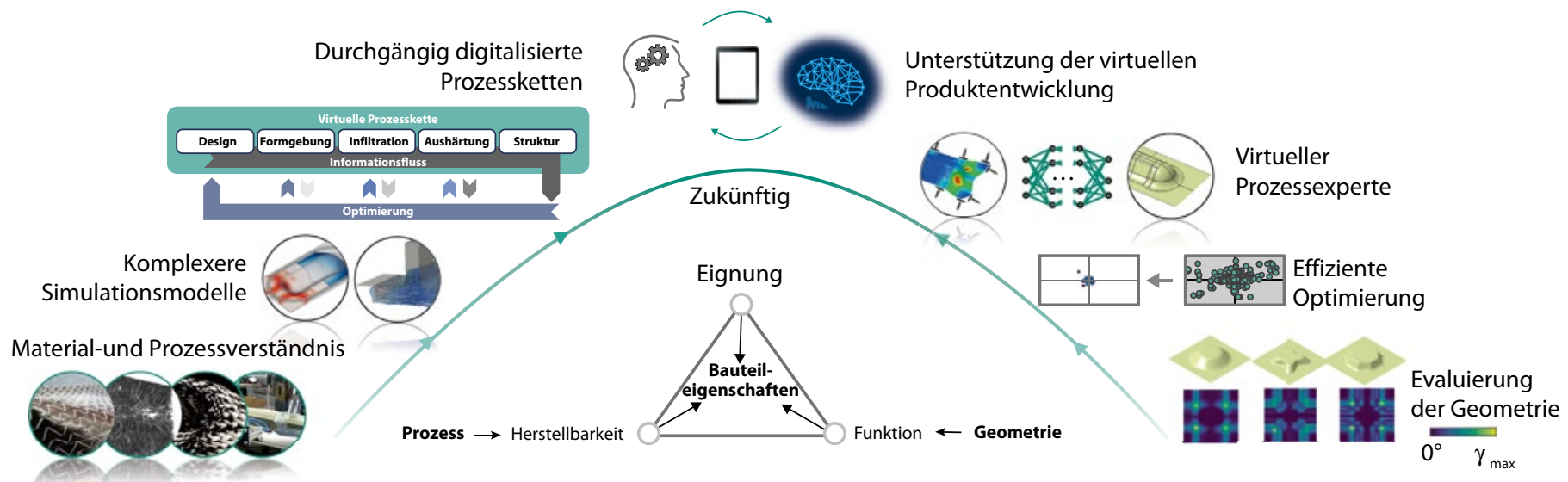

Bild 8 Zukünftige, KI-unterstützte durchgängig digitalisierte Produktentwicklung (๑ KIT|FAST) 
Geschäftsfelder ab, beispielsweise cloudbasierte KI-Engineeringdienstleistungen.

Diese Methoden werden in den kommenden zehn Jahren einen wachsenden Stellenwert innerhalb von Entwicklungsprozessen einnehmen und neue Tools bereitstellen, Bild 8 , um den Ingenieur von morgen bei seiner Arbeit zu unterstützen. KI-Methoden werden dabei numerische Simulation nicht ersetzen, sondern sinnvoll ergänzen und ihre Zugänglichkeit erhöhen.

\section{Quellen}

[1] Henning, F.; Kärger, L.; Dörr, D.; Schirmaier, F. J. Seuffert, J.; Bernath, A.: Fast processing and continuous simulation of automotive structural composite components. In: Composites Science and Technology 171 (2019), S. 261-279

[2] Kärger L.Bernath, A.Fritz, F:Galkin, S: Magagnato, D: Oeckerath, A. Schön, A: Henning, F: Development and validation of a CAE chain for unidirectional fibre reinforced composite components, In: Compos. Struct. 132 (2015): S. 350-358

[3] Meyer, N.; Schöttl, L.; Bretz, L.; Hrymak, A.; Kärger, L.: Process Simulation of Sheet Molding Compound
(SMC) using Direct Bundle Simulation. International Conference of Composites Science and Technology (ICCST/12), Sorrento/Italy, 2019

[4] Poppe, C.; Dörr, D.; Henning, F.; Kärger, L.: A 2D modeling approach for fluid propagation during FEforming simulation of continuously reinforced composites in wet compression moulding. AlP Conference Proceedings 1960, Palermo/Italy, 2018

[5] Poppe C.; Rosenkranz T; Dörr, D.; Kärger, L.: Comparative experimental and numerical analysis of bending behaviour of dry and low viscous infiltrated woven fabrics. In: Composite Part A 124 (2019)

[6] Galkin, S.; Kunze, E.; Kärger, L.; Böhm, R.; Gude, M: Experimental and Numerical Determination of the Local Fiber Volume Content of Unidirectional Non-Crimp Fabrics with Forming Effects. In: Composite Sciences 19 (2019), Nr. 3

[7] Bell, J.: Machine Learning: Hands-On for Developers and Technical Professionals. Indianapolis: Wiley, 2015

[8] Pfrommer, J.; Zimmerling, C.; Liu, J.; Kärger, L.; Henning, F.; Beyerer, J.: Optimisation of manufacturing process parameters using deep neural networks as surrogate models, Procedia CIRP 72, 2018

[9] Zimmerling, C.; Trippe, D.; Fengler, B.; Kärger, L.: An Approach for Rapid Prediction of Textile Draping Results for Variable Composite Component Geometries Using Deep Neural Networks. In: AIP-Proceedings ESAFORM 2019, Vitoria-Gasteiz, 2019

[10] Zimmerling, C.; Dörr, D.; Henning, F.; Kärger, L.: A Machine Learning Assisted Approach for Textile
Formability Assessment and Design Improvement of Composite Components. In: Composites Part A 124 (2019)

[11] Autodesk GmbH. Online: www.autodesk.de/solutions/generative-design, aufgerufen am 01.09.2019

\section{Danke}

Die vorgestellten Ergebnisse stammen aus den Projekten „Forschungsbrücke", gefördert durch das Landesministerium MWK Baden-Württemberg, dem DFG-Graduiertenkolleg GRK 2078, dem ITEA-Projekt VMAP, sowie dem DFG-Forschungsprojekt KA 4224/1-1. Sie sind ebenfalls Teil der YoungInvestigator-Group (YIG) "Green Mobility", gefördert durch die Vector-Stiftung. Die Autoren danken für die Förderung und Unterstützung. 


\section{Repository KITopen}

Dies ist ein Postprint/begutachtetes Manuskript.

Empfohlene Zitierung:

Zimmerling, C.; Poppe, C.; Kärger, L.

Virtuelle Produktentwicklung mittels Simulationsmethoden und KI. 2019. Lightweight design, 12 (6)

doi:10.5445/IR/1000124621

Zitierung der Originalveröffentlichung:

Zimmerling, C.; Poppe, C.; Kärger, L.

Virtuelle Produktentwicklung mittels Simulationsmethoden und KI. 2019. Lightweight design, 12 (6), 12-19.

doi:10.1007/s35725-019-0069-8 